\title{
Investigating energy dissipation through nucleon transfer reactions
}

\author{
D.C. Rafferty ${ }^{1, a}$, M. Dasgupta ${ }^{1}$, D.J. Hinde ${ }^{1}$, C. Simenel ${ }^{1}$, K. J. Cook ${ }^{1}$, I. P. Carter ${ }^{1}$, D. H. Luong ${ }^{1}$, S. D. McNeil ${ }^{1}$, \\ K. Ramachandran ${ }^{1 \mathrm{~b}}$, A. Wakhle ${ }^{1 \mathrm{c}}$, and E. Williams ${ }^{1}$ \\ ${ }^{1}$ Department of Nuclear Physics, Australian National University, Canberra, Australia
}

\begin{abstract}
Nucleon and cluster transfer probabilities have been measured in the collisions of ${ }^{16,18} \mathrm{O},{ }^{19} \mathrm{~F}$ with ${ }^{204,208} \mathrm{~Pb},{ }^{209} \mathrm{Bi}$ for charge stripping channels down to $\Delta \mathrm{Z}=-3$. Strong evidence of correlated nucleon transfer has been observed in particular channels, and neighbouring systems are seen to differ significantly in their behaviour. New measurements were made using an improved $\Delta \mathrm{E}-\mathrm{E}$ telescope. The back-scattered projectilelike fragments were measured in the telescope at $\theta_{l a b}=160.6^{\circ}$, and in combination with monitor detectors at forward angles allowed determination of absolute transfer probabilities. The improved design allows isotopic yields to be measured with greater precision.
\end{abstract}

\section{Introduction}

Recent advances in experimental techniques have allowed measurement of fusion cross sections at low energies with unprecedented precision [1], opening up the deep sub-barrier regime to examination. Fusion cross sections have been seen to fall off much faster with energy than predicted by standard Coupled Channels (CC) approaches to calculate them below a certain threshold [2,3], calling into doubt the efficacy of these models in this regime [4]. An abundance of recent measurements has shown this hindrance effect to be present in a wide variety of systems [5-8], such that it appears to be a general feature of heavy ion fusion. Importantly, this may affect fusion reactions of astrophysical importance [9, 10], suggesting important consequences for stellar evolution.

The physical origin of this phenomenon has been extensively sought [11-13], but no convincing explanation capable of consistently describing fusion above and below the Coulomb barrier has been forthcoming. Several theoretical explanations have been put forward- Amongst other mechanisms, energy dissipation [14, 15] and transfer processes [16] in heavy ion collisions have been suggested to play a part in the observed suppression of fusion cross sections in many systems below the Coulomb barrier. The inclusion of dissipative couplings in fusion models has been shown to potentially account for the hindrance effect [14]. It is therefore of interest to study how energy is dissipated in low-energy transfer reactions, and particularly how this differs depending on the transfer process in question.

\footnotetext{
a e-mail: dominic.rafferty@anu.edu.au

${ }^{b}$ Present address: Nuclear Science Division, Bhaba Atomic Research Centre, Mumbai, India

${ }^{\mathrm{c}}$ Present address: National Superconducting Cyclotron Laboratory, Michigan State University,East Lansing, Michigan, USA
}

In this work we report measurements of transfer probabilities in the systems ${ }^{16} \mathrm{O}+{ }^{208} \mathrm{~Pb},{ }^{18} \mathrm{O}+{ }^{208} \mathrm{~Pb},{ }^{19} \mathrm{~F}+{ }^{208} \mathrm{~Pb}$, ${ }^{16} \mathrm{O}+{ }^{209} \mathrm{Bi}$ and ${ }^{18} \mathrm{O}+{ }^{204} \mathrm{~Pb}$.

\section{Experiment}

Measurements were taken at the ANU 14UD Heavy Ion Accelerator Facility, where beams of ${ }^{12,13} \mathrm{C},{ }^{16,18} \mathrm{O}$ and ${ }^{19} \mathrm{~F}$ were produced in the energy range $3-5 \mathrm{MeV} / \mathrm{A}$, corresponding to $90-100 \%$ of the Coulomb barrier energy $\mathrm{V}_{B}$ in the colliding systems. Back-scattered projectile-like fragments were detected at a laboratory angle $\theta_{\text {lab }}=$ $160.6^{\circ}$ using an improved $\Delta \mathrm{E}-\mathrm{E}$ telescope, with yields normalised to Rutherford scattering events in two forward angle monitor detectors at $\theta_{l a b}= \pm 22.5^{\circ}$ to give absolute probabilities of the identified channels. A schematic of the target chamber is shown in figure 1.

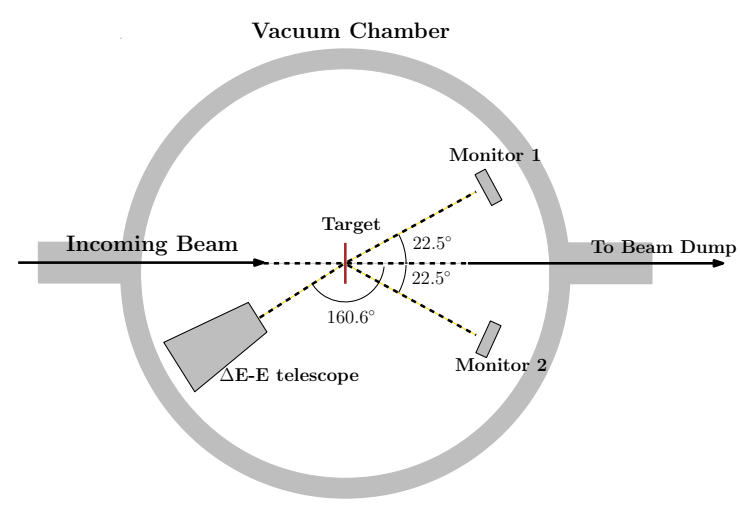

Figure 1. Schematic diagram of experimental setup. 
The $\Delta \mathrm{E}-\mathrm{E}$ telescope used in this experiment was an improved design of one used in previous experiments at the ANU, consisting of a Frisch grid ion chamber system [17] coupled with a Micron Semiconductor ion-implanted Silicon detector. The design of the detector is depicted in figures 2 and 3 . The grid is biased at an intermediate potential between that of the anode and cathode, and allows passage of the electrons liberated along the ionising path of the incoming particle, which are drawn towards the anode. Figure 3 shows the electrical design of the gridded ion-chamber. The signal is measured across the resistor $\mathrm{R}$ between the grid and anode, and since this depends only on the fast drift of liberated electrons and not the slower positively charged ionic Propane molecules, the time constant of the circuit can be set to be much smaller than the basic ion chamber. As such the grid system can handle high count rates.

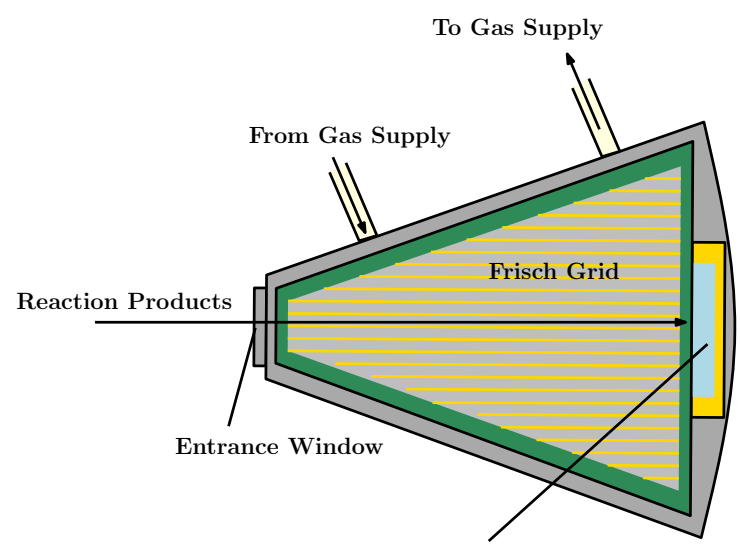

ORTEC Surface-Barrier Si Detector

Figure 2. Top-down schematic view of $\Delta \mathrm{E}$-E telescope.

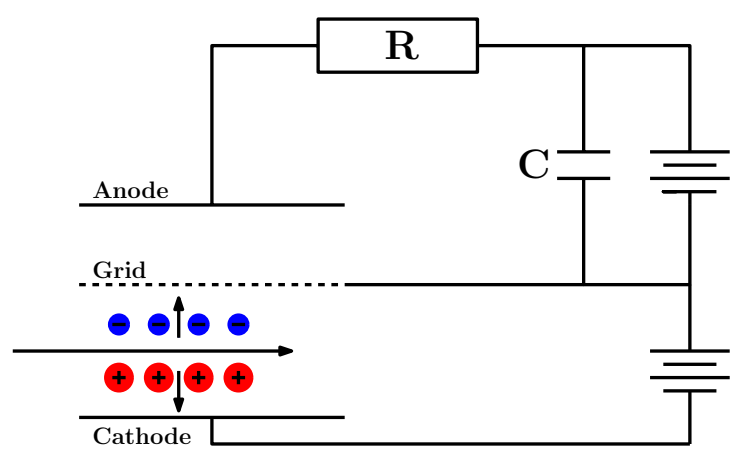

Figure 3. Electronic design of Frisch grid ion chamber system. Blue circles represent liberated electrons, red circles indicate remaining positively charged ions after passage of the incoming ion. Adapted from [17].

The new design incorporated side electrodes in the ion chamber, acting as a potential divider between the cathode and grid, ensuring the electric field within the chamber be as uniform as possible to maximise uniformity of charge collection. The grid wires were of uniform $1 \mathrm{~mm}$ spacing and $20 \mu \mathrm{m}$ thick, providing high transparency to the pass-

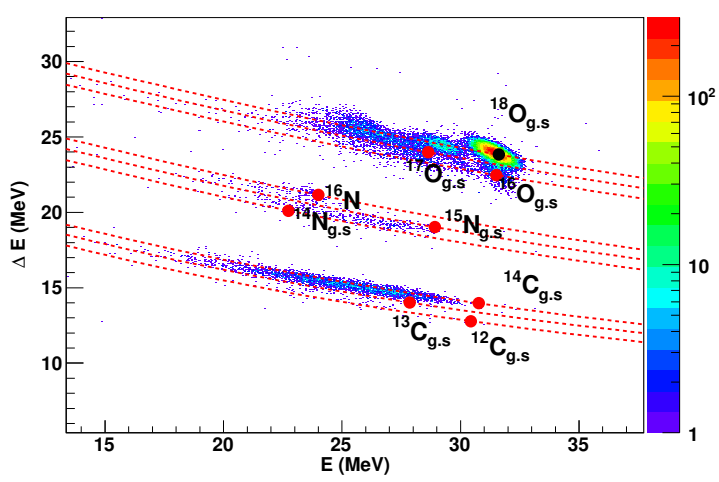

Figure 4. Calibrated $\Delta \mathrm{E}-\mathrm{E}$ spectrum obtained from ${ }^{18} \mathrm{O}+{ }^{208} \mathrm{~Pb}$ at $0.99 \mathrm{~V}_{B}$. Dashed red lines indicate expected tracks (calculated using SRIM [18]). Black dot indicates expected elastic peak position. Red dots indicate expected ground-state-to-ground-state transfer peak positions. As expected from equation 1, products are well separated in charge, with finer features indicating the presence of different isotopes.

ing electrons.

In traversing matter characterised by atomic number $\mathrm{Z}$ and mass $A$, an ion of charge $Z_{p}$ and mass $A_{p}$ with energy $E$ will lose energy according to the Bethe-Bloch formula:

$$
-\frac{d E}{d x} \propto \frac{Z_{p}^{2} A_{p}}{E} \frac{Z}{A}
$$

Where $\mathrm{E}$ is the projectile energy, with $\mathrm{Z}$ and $\mathrm{A}$ being the proton and atomic numbers characterising the matter in which the projectile is travelling. Projectiles are thus expected to lose energy at a rate which is linearly proportional to their mass, and quadratically proportional to their ionic charge. The working principle of the $\Delta \mathrm{E}-\mathrm{E}$ telescope is thus to identify incident ions from their unique locus in the obtained $\Delta \mathrm{E}$-E spectrum.

\section{Analysis}

\subsection{Separation of products}

The main challenge in identifying reaction products is in identifying the unique track in the $\Delta \mathrm{E}-\mathrm{E}$ spectrum belonging to each isotope. For this purpose, spectra were recorded of scattering of projectiles corresponding to the products of interest from a thick Tantalum target. The projectile can scatter at any depth in the target, and thus emerge over a wide energy range as evident in figure 5 . This measurement should occur at energies far below barrier to ensure that transfer is minimal, and consequently that nearly all events seen in the detector correspond to the beam species. An algorithm was then used to fit projections of each bin in the $\mathrm{E}$ axis (corresponding to the signal in the back Silicon detector in the telescope) onto the $\Delta \mathrm{E}$ axis over the band of events, finding the centroid of events in that bin, and stitching these together to generate a graph over the spectra corresponding to that isotope's unique locus. Due to the discrete nature of the data, the resulting graph can be very noisy- this is eliminated 
within the algorithm by making use of a moving average calculation.

After application of the aforementioned algorithm to define the track in this way, the graph can be extended by finding the elastic peak position of all thin target scattering, and stitching these onto the already obtained track graph. Due to the discrete nature of the data a smoothing algorithm is applied to eliminate discontinuities in the graph.

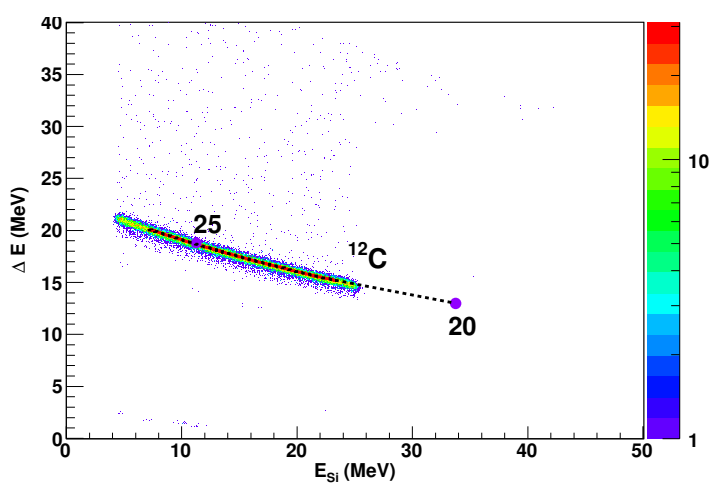

Figure 5. Identification of ${ }^{12} \mathrm{C}$ track in $\Delta \mathrm{E}-\mathrm{E}$ spectrum. Numbers are run identifiers. Pink dots indicate the fitted elastic peak position in those runs. Run 20 is ${ }^{12} \mathrm{C}+{ }^{197} \mathrm{Au}$ at $1.05 \mathrm{~V}_{B}$. Run 25 is ${ }^{12} \mathrm{C}+{ }^{197} \mathrm{Au}$ at $0.67 \mathrm{~V}_{B}$. The same procedure was used to identify the tracks of ${ }^{16,18} \mathrm{O}$ and ${ }^{19} \mathrm{~F}$.

Once the tracks have been identified, isotopes within each band can be separated by finding the relative energy loss in the ionisation chamber of all events relative to the nearest identified track. An example is shown in figure 6, where the identified locus of ${ }^{12} \mathrm{C}$ is plotted over one of the measured $\Delta \mathrm{E}-\mathrm{E}$ spectra. The resulting distribution is then fitted with multiple Gaussian functions, as shown in figure 7 , and the yields of each product found by taking the integrals of the individually fitted Gaussian components. The associated uncertainty in these yields is taken as that arising from the fitting process. Once the yields have been determined, they are normalised to Rutherford scattering events in the forward angle monitor detectors, giving absolute probabilities for each reaction channel.

\subsection{Q-value analysis}

In order to examine energy dissipation, it is necessary to obtain the Q-value spectrum of the reaction products, which requires gates to be defined on events corresponding to each isotope of interest. This is achieved by defining the gate limits in the $\Delta \mathrm{E}$ axis to correspond to the intersections between adjacent Gaussian components in the relative energy loss spectrum. By defining the limits in such a way, the events in the spectrum are attributed to different isotopes on the basis of their most probable identity. The gate limits in the $\mathrm{E}_{\mathrm{Si}}$ axis are then defined by the expected ground-state-to-ground-state peak positions as shown in figure 4 , taking into consideration the width of the elastic peak.

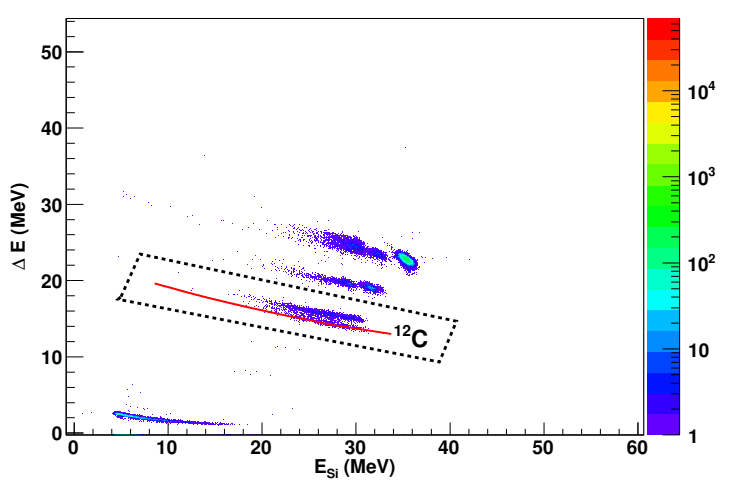

Figure 6. Red line indicates the identified ${ }^{12} \mathrm{C}$ track plotted on the $\Delta \mathrm{E}-\mathrm{E}$ spectrum obtained from ${ }^{16} \mathrm{O}+{ }^{208} \mathrm{~Pb}$ at $0.99 \mathrm{~V}_{B}$. Black dashed line indicates a gate selecting the events to be added to the relative energy loss spectrum (see figure 7).

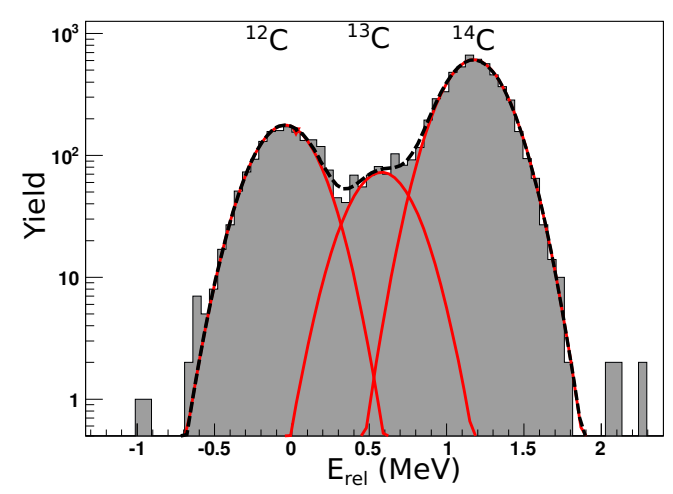

Figure 7. The resulting relative energy loss spectrum obtained of Carbon events produced in the reaction ${ }^{16} \mathrm{O}+{ }^{208} \mathrm{~Pb}$ at $0.99 \mathrm{~V}_{B}$. Red curve shows the multiple Gaussian function fitted to the distribution. Green curves indicate the resulting fitted components which are attributed to yields of the expected isotopes, in this case ${ }^{12,13,14} \mathrm{C}$. Red dashed lines indicate the determined peak positions of each isotope. Blue dashed lines indicate the limits of the gates defined around each isotope.

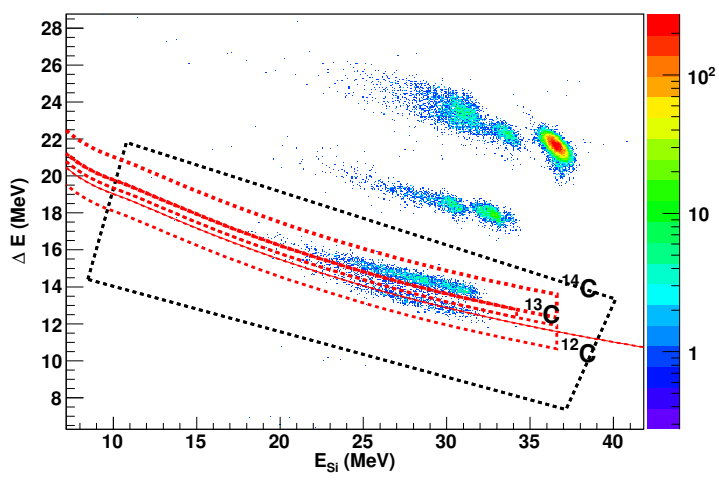

Figure 8. Red dashed lines indicate the gates defined to identify different isotopes in the $\Delta \mathrm{E}-\mathrm{E}$ spectrum. The solid red line shows the identified track of ${ }^{12} \mathrm{C}$, from which the relative energy loss spectrum was determined (see text). 
Once gates have been defined around the reaction products of interest, an event-by-event analysis is conducted in which all events are traced back to the center-ofmomentum frame of the original reaction, and the Q-value reconstructed according to:

$$
Q=\frac{A_{3}+A_{4}}{A_{4}} E_{3}+\frac{A_{4}-A_{1}}{A_{4}} E_{1}-\frac{2 \sqrt{A_{1} A_{3} E_{1} E_{3}}}{A_{4}} \cos \theta_{\text {lab }} .
$$

Where the subscripts $1,2,3,4$ indicate the projectile, target, projectile-like fragment and target-like fragment respectively. Excitation energies are then determined from:

$$
E^{*}(\mathrm{MeV})=Q-Q_{g . s} .
$$

Where $\mathrm{Q}_{g . s}$ is the ground-state-to-ground-state transfer Q-value.

\subsection{Correlated vs. Sequential Nucleon Transfer}

In the simplest semiclassical models of multinucleon transfer, nucleons are expected to be transferred sequentially. In this limit it should be expected that the probability for 2 nucleon transfer be the square of that for 1 nucleon transfer:

$$
P_{2 n}=\left(P_{1 n}\right)^{2} .
$$

Deviations from this behaviour provides an indication of a contribution of correlated transfer to the reaction channel in question [19]. Note that fully microscopic calculations using e.g. Time Dependent Hartree-Fock theory show that this expression serves as only an approximation to multinucleon transfer $[20,21]$.

In the semiclassical approximation, at low energies (or equivalently large internuclear distances) where the overlap between nuclei is minimal, the probability for nucleon transfer is given by [22]:

$$
P_{t r} \propto \exp \left(-2 \alpha r_{\min }\right)
$$

Where $\alpha$ is known as the slope parameter, and is dependent on the binding energy of the transferred particle in the donor nucleus:

$$
\alpha=\frac{\sqrt{2 \mu E_{b}}}{\hbar}
$$

Where $\mu$ is the reduced mass of the particle and $\mathrm{E}_{b}$ its binding energy. $r_{\text {min }}$ is the distance of closest approach of the colliding system, defined as the distance between the centres of the two nuclei. In the low energy limit, where the two nuclei do not come into close enough contact for the strong nuclear forces to take effect, the trajectories can be considered to be purely a result of the repulsive Coulomb force, and $r_{\min }$ is then given by the simple expression:

$$
r_{\text {min }}^{\text {Coul }}=\frac{Z_{p} Z_{t} e^{2}}{4 \pi \epsilon_{0}} \frac{1}{2 E_{C M}}\left(1+\operatorname{cosec} \frac{\theta_{C M}}{2}\right)
$$

Where the subscripts $\mathrm{p}$ and $\mathrm{t}$ indicate the projectile and target respectively.

\section{Results and Discussion}

In the discussion that follows, all transfer results reported are those of the stripping type- where nucleons are transferred from the projectile to the target.

An examination of charge transfer probabilities in the ${ }^{18} \mathrm{O}+{ }^{208} \mathrm{~Pb}$ reaction shows a clear signature of correlated nucleon transfer. The $\Delta \mathrm{Z}=-2$ channel is more probable than the $\Delta \mathrm{Z}=-1$ channel over the entire energy range considered here. A closer examination of the Carbon isotopes produced in this reaction suggests that the dominant contribution is due to transfer of an alpha particle, with the vast majority of these events being identified with ${ }^{14} \mathrm{C}$, as shown in figure 10.

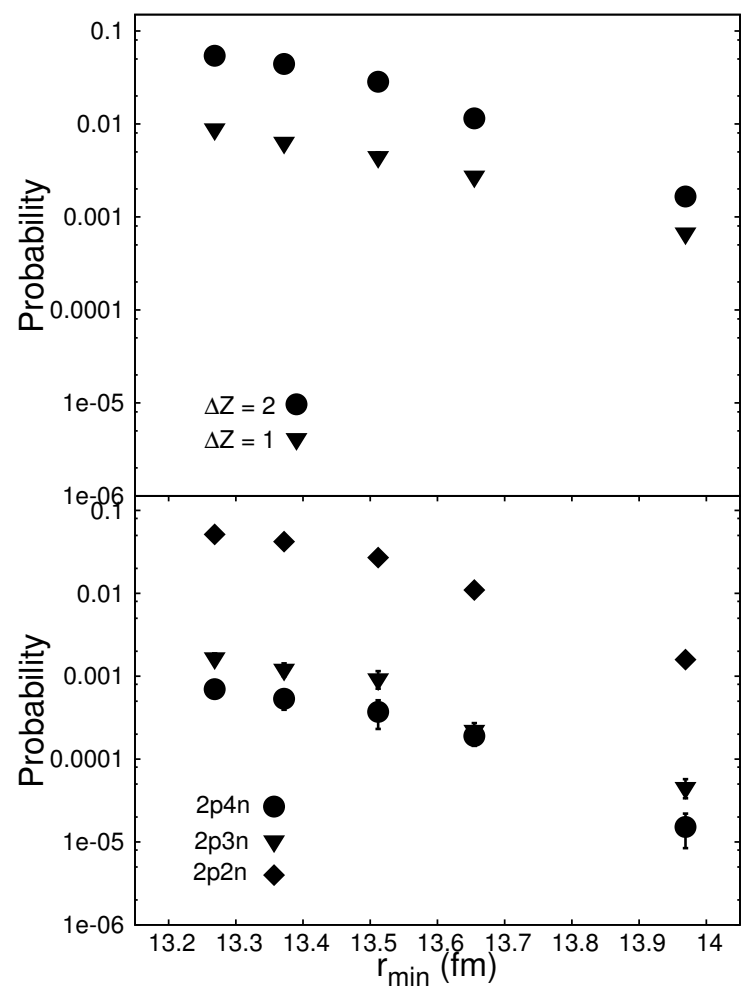

Figure 9. Transfer channels in ${ }^{18} \mathrm{O}+{ }^{208} \mathrm{~Pb}$ : Top panel shows charge transfer $(\Delta \mathrm{Z})$ channels. Bottom panel shows isotopic contributions to Carbon products $(\Delta \mathrm{Z}=2)$.

This result is very similar to that obtained in the ${ }^{18} \mathrm{O}+$ ${ }^{204} \mathrm{~Pb}$ reaction (as seen in figure 10 ), supporting the idea that the importance of this channel is due to the intrinsic properties of the ${ }^{18} \mathrm{O}$ nucleus. This is an interesting result when compared with the results of the ${ }^{16} \mathrm{O}+{ }^{208} \mathrm{~Pb}$ reaction, which is shown in figure 11 . Here contributions from correlated $2 p$ transfer and alpha transfer are both evident, though with probabilities lower than the observed $1 \mathrm{p}$ transfer, and with the $2 \mathrm{p}$ channel dominant over the $\Delta \mathrm{Z}=2$ channel. ${ }^{16} \mathrm{O}$ has traditionally been thought to be a good candidate for alpha clustering structures, being a light $\mathrm{N}=\mathrm{Z}$ nucleus, though this result, as well as those reported in [15], contradict this picture. However, as seen in figure 11, at the largest internuclear separations the $2 \mathrm{p}$ and $\alpha$ transfer channels appear to become almost equivalent. This suggests that at lower energies the alpha 


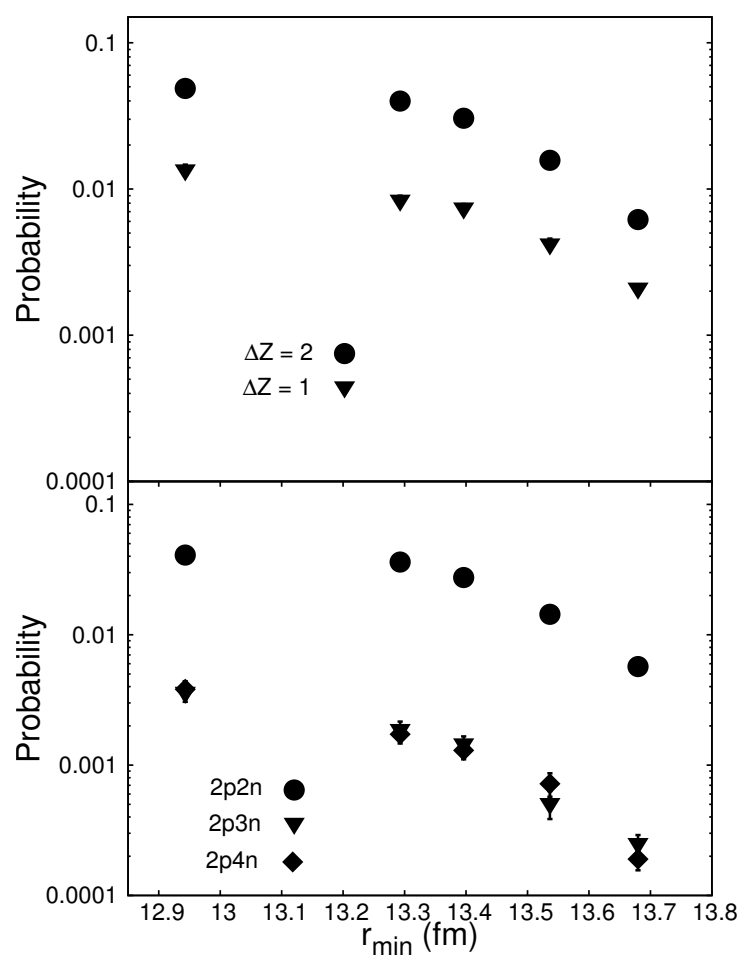

Figure 10. Transfer channels in ${ }^{18} \mathrm{O}+{ }^{204} \mathrm{~Pb}$ : Top panel shows charge transfer $(\Delta Z)$ channels. Bottom panel shows isotopic contributions to Carbon products $(\Delta \mathrm{Z}=2)$.

cluster-structure states may become more important. This may have interesting implications for astrophysics, since Oxygen is important in later stages of lifetimes of high mass stars. A comparison with the data in the ${ }^{16} \mathrm{O}+{ }^{209} \mathrm{Bi}$ reaction (shown in figure 12) shows similar behaviour, with the $2 p$ channel again the dominant $\Delta \mathrm{Z}=-2$ channel, though with an appreciable contribution from the $2 \mathrm{p} 2 \mathrm{n}$ channel.

An examination of the ${ }^{19} \mathrm{~F}+{ }^{208} \mathrm{~Pb}$ (figure 13) reaction again shows strong evidence of cluster transfer. The $\Delta \mathrm{Z}$ $=-1$ channel is by far dominant over the energy range considered, but interestingly, the $\Delta \mathrm{Z}=-2$ and $\Delta \mathrm{Z}=-3$ channels are similar at energies close to but below the barrier, with the $\Delta \mathrm{Z}=-3$ channel dominant at around $0.97 \mathrm{~V}_{B}$, beyond which the $\Delta \mathrm{Z}=-3$ channel falls off much faster than $\Delta Z=-2$. Again, this strongly suggests a contribution from transfer of multinucleon clusters from ${ }^{19} \mathrm{~F}$ to ${ }^{208} \mathrm{~Pb}$. The exact mechanism giving rise to this behaviour is difficult to extract from this experiment. However, with the $\Delta \mathrm{Z}=-2,-3$ channels being around an order of magnitude lower in probability than the $\Delta \mathrm{Z}$ $=-1$ channel suggests that this could be the result of a sequential process in which a proton is first transferred from the ${ }^{19} \mathrm{~F}$, followed by an alpha from the remaining ${ }^{18} \mathrm{O}$ nucleus. The fact that the majority of the Carbon products detected are identified as being ${ }^{14} \mathrm{C}$ seems to support this, and also that similar behaviour is observed for the reactions of ${ }^{18} \mathrm{O}$ on ${ }^{208} \mathrm{~Pb}$ and ${ }^{209} \mathrm{Bi}$. This could, however, alternatively be explained by a contribution from

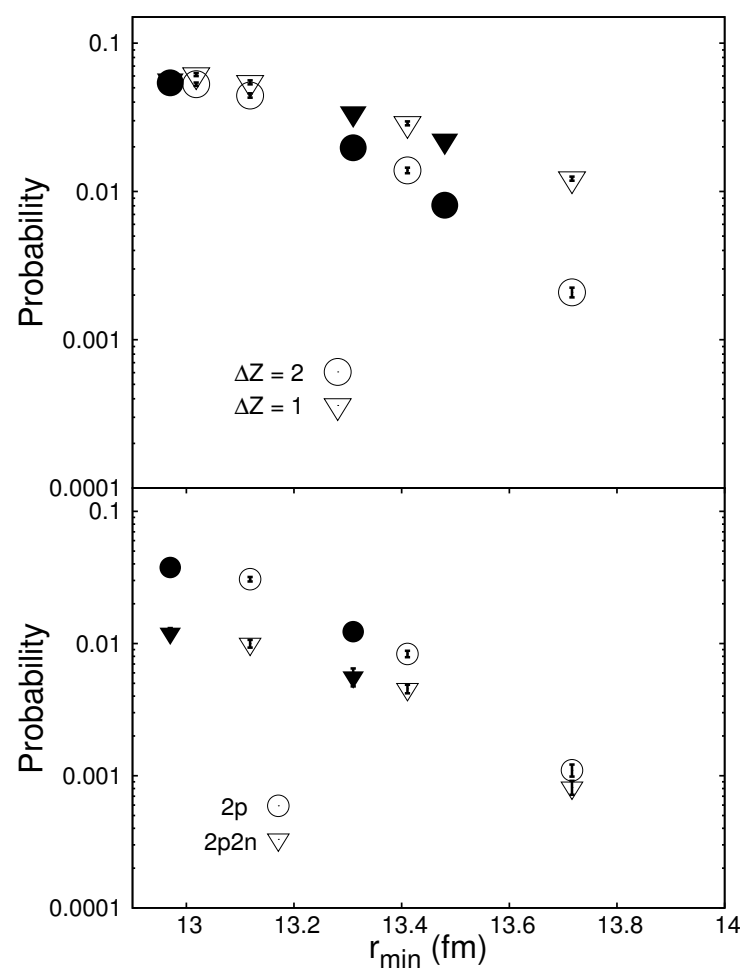

Figure 11. Transfer channels in ${ }^{16} \mathrm{O}+{ }^{208} \mathrm{~Pb}$ : Top panel shows charge transfer $(\Delta \mathrm{Z})$ channels. Bottom panel shows isotopic contributions to Carbon products $(\Delta Z=2)$. Empty symbols represent measurements taken in the present work. Filled symbols are taken from [15].

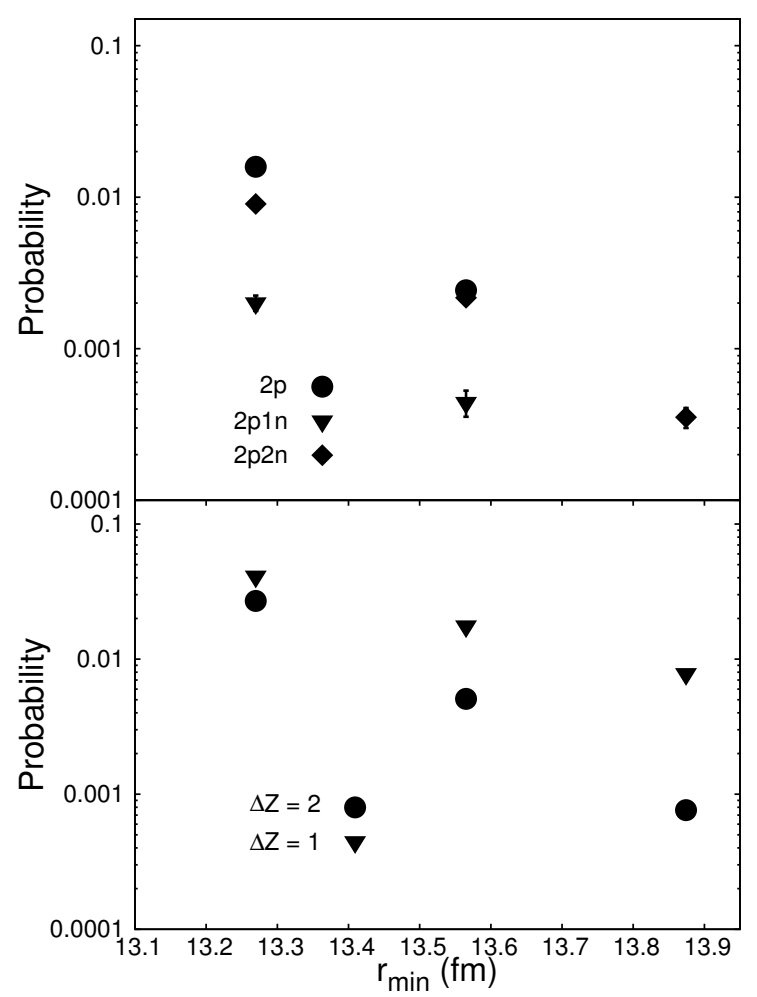

Figure 12. Transfer channels in ${ }^{16} \mathrm{O}+{ }^{209} \mathrm{Bi}$ : Top panel shows charge transfer $(\Delta \mathrm{Z})$ channels. Bottom panel shows isotopic contributions to Carbon products $(\Delta \mathrm{Z}=2)$. 
correlated $\mathrm{p} \alpha$ cluster transfer. This result was previously seen also in the reaction ${ }^{19} \mathrm{~F}+{ }^{232} \mathrm{Th}$ at near-barrrier energies as reported in Ref. [23].

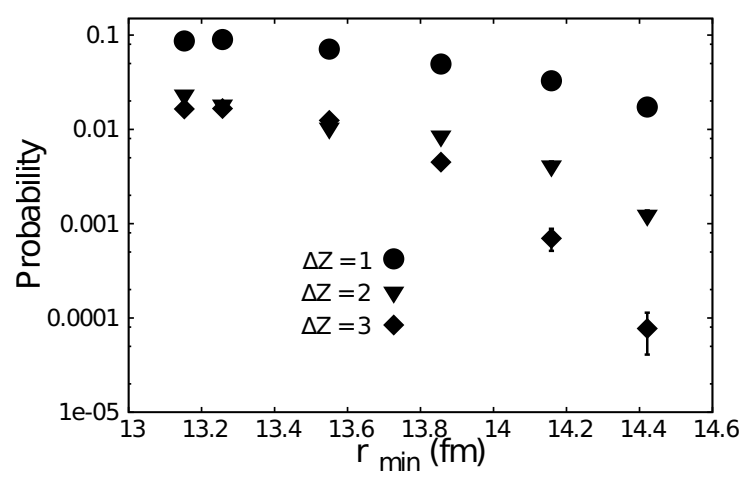

Figure 13. $\Delta \mathrm{Z}$ transfer channels in ${ }^{19} \mathrm{~F}+{ }^{208} \mathrm{~Pb}$.

Figures 14 and 15 show the excitation spectra of the reaction products in the ${ }^{12} \mathrm{C}$ and ${ }^{14} \mathrm{C}$, in which it is evident that the reaction products can be produced at high excitation energies. The ground state is seen to be very weakly populated in the products of these reactions, with excited states populated up to tens of $\mathrm{MeV}$ even at energies far below the Coulomb barrier. These results support the importance of including dissipative couplings generated by cluster transfer in fusion models, which may prove important to describing the fusion hindrance effects $[4,9]$ that are seen at low energies.

\section{Conclusions and Outlook}

The present work has demonstrated the vastly differing low energy transfer mechanisms amongst systems in the region of the ${ }^{16} \mathrm{O}+{ }^{208} \mathrm{~Pb}$ system, showing that clustering properties in light nuclei are of great importance when considering the dynamics of nuclear reactions in the deep sub-barrier regime. Also present is evidence supporting the importance of dissipative effects in low energy reactions, which are not presently accounted for within the Coupled Channels paradigm for predicting fusion cross sections.

Of particular interest would be to examine how the degree of dissipation varies depending on the particular reaction mechanism leading to the product in question. In the present experiment it is not possible to distinguish between one-step and sequential processes on an event-byevent basis, and thus, for example, it cannot be determined whether the small number of events found to correspond to ground-state-to-ground-state transfer are the result of one or the other.

Further work in the ANU reaction dynamics group is planned to explore these effects in more detail, and amongst other reacting systems, to better understand how they should be treated within a dynamical model of fusion.

\section{Acknowledgements}

This work was supported by Australian Research Council grants FT120100760 and FL110100098. We wish to acknowledge the support of the technical staff at the ANU Heavy Ion Accelerator Facility for their assistance in preparation and smooth operation of the experiment. Thanks also to Dr M. Evers for discussions.

\section{References}

[1] M. Dasgupta, D. Hinde, N. Rowley, A. Stefanini, Annual Review of Nuclear and Particle Science 48, 401 (1998)

[2] C. Shen, D. Boilley, Q. Li, J. Shen, Y. Abe, Physical Review C 83, 054620 (2011)

[3] T. Ichikawa, K. Hagino, A. Iwamoto, Physical Review C 75, 064612 (2007)

[4] M. Dasgupta, D. Hinde, A. Diaz-Torres, B. Bouriquet, C.I. Low, G.J. Milburn, J. Newton, Physical review letters 99, 192701 (2007)

[5] J. Khuyagbaatar, K. Nishio, S. Hofmann, D. Ackermann, M. Block, S. Heinz, F. Heßberger, K. Hirose, H. Ikezoe, B. Kinlder et al., Physical Review C 86, 064602 (2012)

[6] H. Esbensen, C. Jiang, A. Stefanini, Physical Review C 82, 054621 (2010)

[7] A. Stefanini, G. Montagnoli, L. Corradi, S. Courtin, E. Fioretto, A. Goasduff, F. Haas, P. Mason, R. Silvestri, P.P. Singh et al., Physical Review C 82, 014614 (2010)

[8] C. Jiang, B. Back, H. Esbensen, J. Greene, R. Janssens, D. Henderson, H. Lee, C. Lister, M. Notani, R. Pardo et al., Physical Review C 78, 017601 (2008)

[9] C. Jiang, K. Rehm, B. Back, R. Janssens, Physical Review C 79, 044601 (2009)

[10] L. Gasques, E. Brown, A. Chieffi, C. Jiang, M. Limongi, C. Rolfs, M. Wiescher, D. Yakovlev, Physical Review C 76, 035802 (2007)

[11] C. Jiang, A. Stefanini, H. Esbensen, K. Rehm, L. Corradi, E. Fioretto, P. Mason, G. Montagnoli, F. Scarlassara, R. Silvestri et al., Physical Review C 82, 041601 (2010)

[12] C. Jiang, K. Rehm, R. Janssens, H. Esbensen, I. Ahmad, B. Back, P. Collon, C. Davids, J. Greene, D. Henderson et al., Physical review letters 93, 012701 (2004)

[13] C. Jiang, B. Back, H. Esbensen, R. Janssens, K. Rehm, Physical Review C 73, 014613 (2006)

[14] A. Diaz-Torres, D. Hinde, M. Dasgupta, G. Milburn, J. Tostevin, Physical Review C 78, 064604 (2008)

[15] M. Evers, M. Dasgupta, D. Hinde, D. Luong, R. Rafiei, R. Du Rietz, C. Simenel, Physical Review C 84, 054614 (2011)

[16] A. Stefanini, G. Montagnoli, H. Esbensen, L. Corradi, S. Courtin, E. Fioretto, A. Goasduff, J. Grebosz, F. Haas, M. Mazzocco et al., Physics Letters B 728, 639 (2014) 


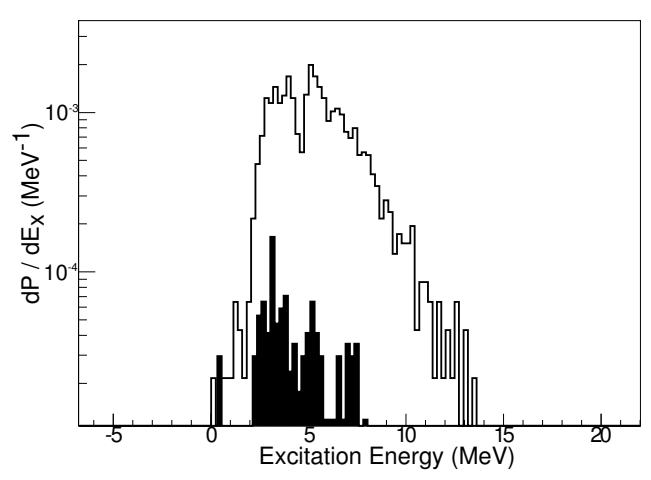

Figure 14. Excitation energy spectrum of ${ }^{14} \mathrm{C}$ products formed in the reaction ${ }^{16} \mathrm{O}+{ }^{208} \mathrm{~Pb}$.

[17] G.F. Knoll, Radiation detection and measurement (John Wiley \& Sons, 2010)

[18] J.F. Ziegler, M. Ziegler, J. Biersack, Nuclear Instruments and Methods in Physics Research Section B: Beam Interactions with Materials and Atoms 268, 1818 (2010)

[19] P. Sahu, R. Choudhury, D. Biswas, B. Nayak, Physical Review C 64, 014609 (2001)

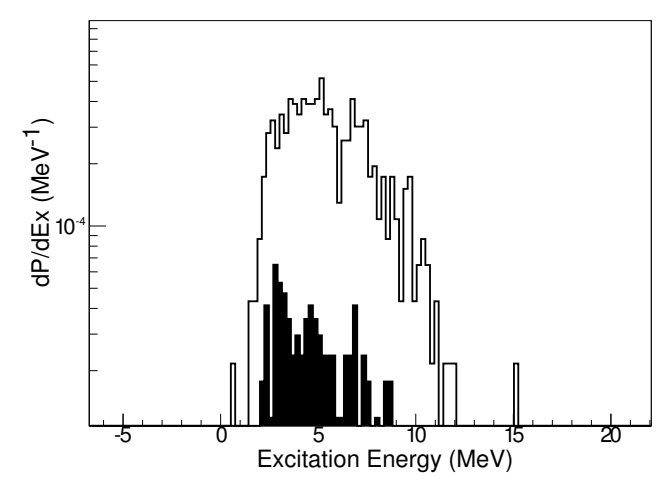

Figure 15. Excitation energy spectrum of ${ }^{12} \mathrm{C}$ products formed in the reaction ${ }^{16} \mathrm{O}+{ }^{208} \mathrm{~Pb}$.

[20] C. Simenel, Physical review letters 105, 192701 (2010)

[21] G. Scamps, D. Lacroix, Physical Review C 87, 014605 (2013)

[22] R. Bass, Nuclear reactions with heavy ions (Springer-Verlag New York, 1980), ISBN 0387096116

[23] D. Biswas, R. Choudhury, B. Nayak, D. Nadkarni, V. Ramamurthy, Physical Review C 56, 1926 (1997) 